\title{
Solitons in one-dimensional nonlinear Schrödinger lattices with a local inhomogeneity
}

\author{
F. Palmero* and R. Carretero-González \\ Nonlinear Dynamical Systems Group, Computational Science Research Center and Department of Mathematics and Statistics, \\ San Diego State University, San Diego, California 92182-7720, USA \\ J. Cuevas \\ Grupo de Física No Lineal, Departamento de Física Aplicada I, EU Politécnica, Universidad de Sevilla, c/Virgen de África s/n, \\ 41011-Sevilla, Spain \\ P. G. Kevrekidis \\ Department of Mathematics and Statistics, University of Massachusetts, Amherst, Massachusetts 01003-4515, USA \\ W. Królikowski \\ Centre for Ultra-high Bandwidth Devices (CUDOS), Nonlinear Physics Centre and Laser Physics Centre, \\ Research School of Physical Science and Engineering, Australian National University, Canberra, ACT 0200, Australia
}

(Received 13 December 2007; published 28 March 2008)

\begin{abstract}
In this paper we analyze the existence, stability, dynamical formation, and mobility properties of localized solutions in a one-dimensional system described by the discrete nonlinear Schrödinger equation with a linear point defect. We consider both attractive and repulsive defects in a focusing lattice. Among our main findings are (a) the destabilization of the on-site mode centered at the defect in the repulsive case, (b) the disappearance of localized modes in the vicinity of the defect due to saddle-node bifurcations for sufficiently strong defects of either type, (c) the decrease of the amplitude formation threshold for attractive and its increase for repulsive defects, and (d) the detailed elucidation as a function of initial speed and defect strength of the different regimes (trapping, trapping and reflection, pure reflection, and pure transmission) of interaction of a moving localized mode with the defect.
\end{abstract}

DOI: 10.1103/PhysRevE.77.036614

PACS number(s): 05.45.Yv, 63.20.Pw, 63.20.Ry

\section{INTRODUCTION}

The past few years have witnessed an explosion of interest in discrete models which has been summarized in a number of recent reviews [1]. This growth has been, to a large extent, motivated by numerous applications of nonlinear dynamical lattice models in areas as broad and diverse as the nonlinear optics of waveguide arrays [2], the dynamics of Bose-Einstein condensates in periodic potentials [3], micromechanical models of cantilever arrays [4], or even simple models of the complex dynamics of the DNA double strand [5]. Arguably, the most prototypical model among the ones that emerge in these settings is the so-called discrete nonlinear Schrödinger equation (DNLS) [6,7]. DNLS may arise as a direct model, as a tight binding approximation, or even as an envelope wave expansion: the DNLS is one of the most ubiquitous models in the nonlinear physics of dispersive discrete systems.

Perhaps the first set of experimental investigations that generated an intense interest in DNLS type equations was in the area of nonlinear optics and, in particular, in fabricated AlGaAs waveguide arrays [8]. In the latter setting a wide range of phenomena such as discrete diffraction, Peierls-

\footnotetext{
*Corresponding author. Permanent address: Nonlinear Physics Group, Escuela Técnica Superior de Ingeniería Informática, Departamento de Física Aplicada I, Universidad de Sevilla, Avda. Reina Mercedes, s/n. 41012-Sevilla, Spain; palmero@us.es
}

Nabarro (PN) barriers (the energetic barrier that a wave needs to overcome to move over a lattice-see details below), diffraction management (the periodic alternation of the diffraction coefficient) $[9,10]$, and gap solitons (structures localized due to nonlinearity in the gap of the underlying linear spectrum) [11] among others [12] were experimentally observed. These phenomena, in turn, led to a large increase also on the theoretical side of the number of studies addressing such effectively discrete media.

A related area where DNLS, although it is not the prototypical model, still yields useful predictions both about the existence and about the stability of nonlinear localized modes is that of optically induced lattices in photorefractive media such as strontium barium niobate (SBN). Since the theoretical inception of such a possibility in Ref. [13], and its experimental realization in Refs. [14-16], there has been an everexpanding growth in the area of nonlinear waves and solitons in such periodic, predominantly two-dimensional, lattices. A wide array of structures has been predicted and experimentally observed in lattices induced with a selffocusing nonlinearity, including, e.g., discrete dipole [17], quadrupole [18], necklace [19], and other multipulse patterns (such as, e.g., soliton stripes [20]), discrete vortices [21], and rotary solitons [22]. Such structures have a definite potential to be used as carriers and conduits for data transmission and processing, in the setting of all-optical communication schemes. A recent review of this direction can be found in Ref. [23] (see also Ref. [24]).

Finally, yet another independent and completely different physical setting where such considerations and structures are 
relevant is that of soft-condensed matter physics, where droplets of the most recently discovered state of matter, namely of Bose-Einstein condensates (BECs), may be trapped in an (egg-carton) optical lattice (OL) potential produced by counterpropagating laser beams in one, two, or even all three directions [25]. The field of BEC has also experienced a huge growth over the past few years, including the prediction and manifestation of modulational instabilities (i.e., the instability of spatially uniform states towards spatially modulated ones) [26], the observation of gap solitons [27], Landau-Zener tunneling (tunneling between different bands of the periodic potential) [28], and Bloch oscillations (for matter waves subject to combined periodic and linear potentials) [29], among many other salient features; reviews of the theoretical and experimental findings in this area have also recently appeared in Ref. [3].

While DNLS combines two important features of many physical lattice systems, namely nonlinearity and periodicity, yet another element which is often physically relevant and rather ubiquitous is disorder. Localized impurities are well known in a variety of settings to introduce not only interesting wave scattering phenomena [30], but also to create the possibility for the excitation of impurity modes, which are spatially localized oscillatory states at the impurity sites [31]. Physical applications of such phenomena arise, e.g., in superconductors [32], in the dynamics of the electron-phonon interactions [33], in the propagation of light in dielectric superlattices with embedded defect layers [34], or in defect modes arising in photonic crystals [35].

In the context of the DNLS, there have been a number of interesting studies in connection to the interplay of the localized modes with impurities. Some of the initial works were either at a quasicontinuum limit (where a variational approximation could also be implemented to examine this interplay) [36] or at a more discrete level but with an impurity in the coupling [37] (see also in the latter setting the more recent studies of a waveguide bend $[38,39]$ and the boundary defect case of Ref. [40]). More recently the experimental investigations of Refs. [41,42] motivated the examinations of linear [43] and nonlinear [43,44] defects in a DNLS context. In the photorefractive context, further recent experimental work has illustrated blocking effects to a probe beam from either bright or dark soliton beams in defocusing waveguide arrays [45].

Our aim in the present work is to systematically examine the properties of the focusing DNLS equation in the presence of both an attractive and a repulsive linear impurity. Our first aim is to present the full bifurcation diagram of the localized modes in the presence of the impurity and how it is drastically modified in comparison to the case of the homogeneous lattice. The relevant bifurcations are quantified whenever possible even analytically, in good agreement with our full numerical computations. A second problem that is examined is that of the threshold for the formation of solitary waves and how it is systematically affected by the presence of impurities both in the repulsive and in the attractive case. This is motivated by the recent examination of the relevant threshold in the homogeneous lattice [46] and its connection with experiments in focusing [47] (and even defocusing [48]) waveguide arrays. Finally, in the same spirit as that of Ref.
[43], but for attractive and repulsive impurities, we systematically investigate the interaction of an incoming solitary wave with the localized impurity, identifying the main observed regimes as being trapping, reflection with trapping, pure reflection, and pure transmission.

This paper is organized as follows. In Sec. II, we introduce the model. In Sec. III, we analyze the existence and stability of localized excitations in a system described by the DNLS with the linear impurity. In Sec. IV, we examine the (energy and/or initial amplitude) threshold for soliton formation. In Sec. V we present our results related to the interaction of moving localized excitations with the impurity and, finally, in Sec. VI, we summarize our findings and present our conclusions.

\section{THE MODEL}

We consider a discrete system with a defect described by the DNLS equation as

$$
i \frac{d \psi_{n}}{d t}+\gamma\left|\psi_{n}\right|^{2} \psi_{n}+C\left(\psi_{n+1}+\psi_{n-1}\right)+\alpha_{n} \psi_{n}=0
$$

where $\psi_{n}$ is the complex field at site $n(n=1 \ldots N) ; \gamma$ is the anharmonicity parameter, $C$ the coupling constant, and parameters $\alpha_{n}$ allow for the existence of local linear inhomogeneities. In this paper, we consider a single point defect, thus $\alpha_{n}=\alpha \delta_{n, n_{0}}$, that can be positive (attractive impurity) or negative (repulsive impurity). In general, the presence of an on-site defect would affect the nearest-neighbor coupling, and Eq. (1) should be modified to take this effect into account, as in Ref. [49]. On the other hand, this inhomogeneity in the coupling can be avoided using different techniques, for example, in nonlinear waveguide arrays, changing slightly the separation between defect waveguide and its nearest neighbors, as it has been done in Ref. [9]. In this work, we will assume that the coupling parameter $C$ is independent on the site and positive.

Upon renormalization of parameters, we consider $\gamma=1$ (focusing case). Note that the defocusing case $(\gamma<0)$ can be reduced, under the staggering transformation $\psi_{n} \rightarrow(-1)^{n} \psi_{n}$, to the previous one with opposite sign of the impurity $\alpha$. Also, under the transformation $\psi_{n} \rightarrow \psi_{n} e^{2 i C t}$, Eq. (1) can be written in the standard form

$$
i \frac{d \psi_{n}}{d t}+\gamma\left|\psi_{n}\right|^{2} \psi_{n}+C \Delta \psi_{n}+\alpha_{n} \psi_{n}=0
$$

where $\Delta \psi_{n}=\psi_{n+1}+\psi_{n-1}-2 \psi_{n}$ is the discrete Laplacian. Throughout this work, we use the form given by Eq. (1).

The DNLS (1) conserves two dynamical invariants, the Hamiltonian

$$
H=-\sum_{n} \frac{1}{2}\left|\psi_{n}\right|^{4}+C\left(\psi_{n}^{*} \psi_{n+1}+\psi_{n}^{*} \psi_{n-1}\right)+\alpha_{n}\left|\psi_{n}\right|^{2},
$$

with canonical variables $q_{n}=\psi_{n}$ and $p_{n}=i \psi_{n}^{*}$, and the (squared $L^{2}$ ) norm or optical power

$$
P=\sum_{n}\left|\psi_{n}\right|^{2}
$$




\section{STATIONARY SOLUTIONS}

In order to study solitons in the system described by Eq. (1), we aim to look for stationary solutions with frequency $\omega$. Thus, substituting

$$
\psi_{n}=e^{i \omega t} \phi_{n}
$$

the stationary analog of Eq. (1) then reads

$$
-\omega \phi_{n}+C\left(\phi_{n+1}+\phi_{n-1}\right)+\phi_{n}^{3}+\alpha_{n} \phi_{n}=0 .
$$

Some of the properties of solitons are related to the existence (or not) and properties of linear localized modes. These modes arise when an inhomogeneity appears and can be obtained from the linearized form (around the trivial solution $\left.\phi_{n}=0, \forall n\right)$ of Eq. (6). In this case, and considering an inhomogeneity located at the first site of the chain and with periodic boundary conditions, the problem reduces to solving the eigenvalue problem

$$
\left[\begin{array}{cccccc}
\alpha & C & 0 & \cdot & \cdot & C \\
C & 0 & C & 0 & \cdot & 0 \\
0 & C & 0 & C & \cdot & \cdot \\
\cdot & \cdot & \cdot & \cdot & \cdot & \cdot \\
\cdot & \cdot & \cdot & C & 0 & C \\
C & . & . & . & C & 0
\end{array}\right]\left[\begin{array}{c}
\phi_{0} \\
\phi_{1} \\
\cdot \\
\cdot \\
\phi_{N-2} \\
\phi_{N-1}
\end{array}\right]=\omega\left[\begin{array}{c}
\phi_{0} \\
\phi_{1} \\
\cdot \\
\cdot \\
\phi_{N-2} \\
\phi_{N-1}
\end{array}\right],
$$

that is a particular case of the eigenvalue problem studied in Ref. [50]. There it was shown that, if $\alpha \neq 0$, the solution corresponds to $N-1$ extended modes and an impurity localized mode. Also, if $N$ becomes large, the frequencies of extended modes are densely distributed in the interval $\Omega$ $\in[-2 C, 2 C]$ and the localized mode can be approximated by the following:

$\alpha>0$ (attractive impurity):

$$
\phi_{n}=\phi_{0}\left[\left(\frac{\alpha}{2 C}+\beta\right)^{-n}+\left(\frac{\alpha}{2 C}+\beta\right)^{n-N}\right]
$$

and

$$
\omega=2 C|\beta|, \quad \beta \equiv \sqrt{\frac{\alpha^{2}}{4 C^{2}}+1},
$$

with an in-phase pattern [see Fig. 1(c)].

$$
\alpha<0 \text { (repulsive impurity): }
$$

$$
\phi_{n}=(-1)^{n} \phi_{0}\left[\left(\frac{\alpha}{2 C}+\beta\right)^{-n}+(-1)^{N}\left(\frac{\alpha}{2 C}+\beta\right)^{n-N}\right]
$$

and

$$
\omega=-2 C|\beta|, \quad \beta \equiv \sqrt{\frac{\alpha^{2}}{4 C^{2}}+1},
$$

with a staggered pattern [see Fig. 1(b)]. In both cases $\phi_{0}$ is an arbitrary constant. In Fig. 1 we depict the linear mode spectrum as a function of the inhomogeneity parameter $\alpha$ [Fig. 1(a)], and examples of the profiles of the ensuing localized modes [Figs. 1(b) and 1(c)].
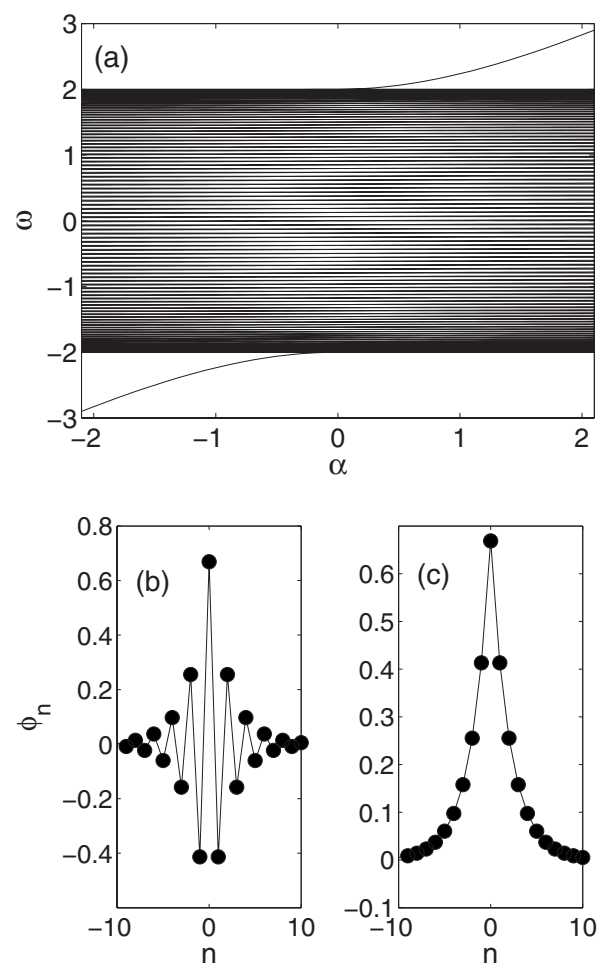

FIG. 1. Linear modes: (a) The dispersion relation as a function of impurity parameter $\alpha$ (notice the impurity mode outside of the interval $[-2 C, 2 C])$. The linear modes are normalized $\left(\Sigma_{n}\left|\phi_{n}\right|^{2}\right.$ $=1$ ), the impurity is located at $n=0$, and periodic boundary conditions are considered. The other panels depict examples of the profiles of the impurity modes. (b) Profile for $\alpha=-1$ (repulsive impurity), and (c) profile for $\alpha=1$ (attractive impurity). In all cases $N$ $=200$ and $C=1$.

In order to explore the existence and stability of the nonlinear stationary states described by Eq. (6), we have used the well-known technique based on the concept of continuation from the anticontinuum (AC) limit using a NewtonRaphson fixed point algorithm [51]. Also, a standard linear stability analysis of these stationary states has been performed, using the ansatz

$$
\phi_{n}=\left\{\phi_{\text {sol }}+\epsilon\left[a_{n} \exp (\lambda t)+b_{n} \exp \left(\lambda^{*} t\right)\right]\right\} \exp (i \omega t),
$$

and solving the ensuing eigenvalue problem. $\phi_{\text {sol }}$ is the solution of Eq. (6) with frequency $\omega, \lambda$ is the linearization eigenvalue, and $\lambda^{*}$ its complex conjugate. Due to symmetries of the system, the eigenvalues appear in quartets (if $\lambda$ is an eigenvalue, so are $\lambda^{*},-\lambda$, and $\left.-\lambda^{*}\right)$. Furthermore, the $U$ (1) invariance of the equation (the so-called phase or gauge invariance) leads to the existence of a pair of zero eigenvalues. If the remaining eigenvalues are imaginary, the state is linearly stable and, on the contrary, the presence of a eigenvalue with a nonzero real part implies instability.

In the homogeneous lattice case of $\alpha=0$, fundamental stationary modes are well known to exist and be centered either on a lattice site or between two adjacent lattice sites [6]. The site-centered solitary waves are always stable, while the intersite centered ones are always unstable [6]. 

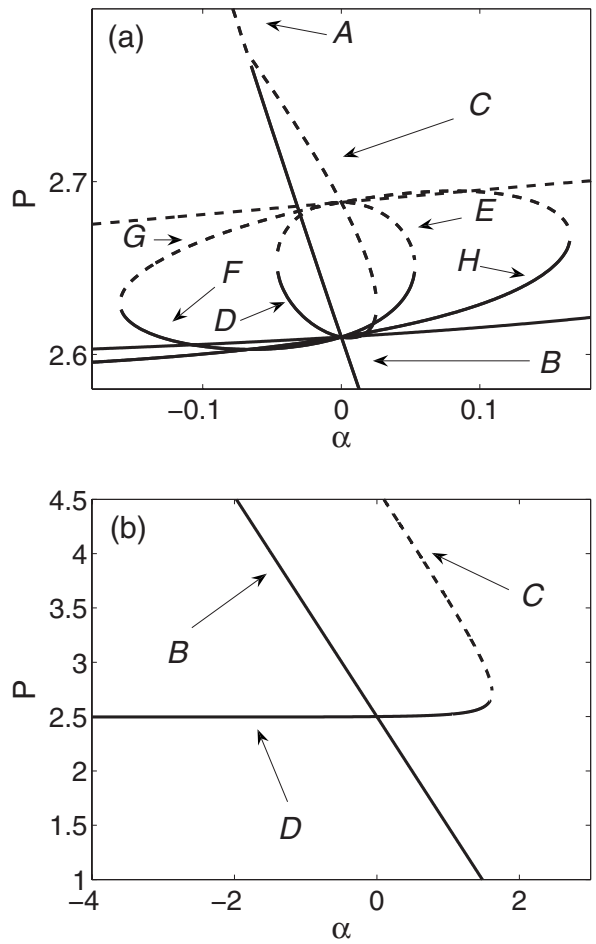

FIG. 2. Bifurcation diagram of stable (solid line) and unstable (dashed line) nonlinear modes. Shown is the power $P$ as a function of the impurity parameter $\alpha$. In all cases $N=100$ and $\omega=2.5$. The panel (a) is for $C=1.0$, while the panel (b) is for $C=0.2$. The branch designation is as follows: $A$ unstable soliton centered at the impurity $\left(n=n_{0}\right), B$ stable on-site soliton centered at $n=n_{0}, C$ unstable intersite soliton centered at $n=n_{0}+0.5, D$ stable on-site soliton at $n=n_{0}+1, E$ unstable intersite soliton at $n=n_{0}+1.5, F$ stable on-site soliton at $n=n_{0}+2, G$ unstable intersite soliton at $n=n_{0}+2.5$, and $H$ stable on-site soliton at $n=n_{0}+3$. The stable on-site mode located at the impurity, in the homogeneous case, disappears for a coupling value of $C \simeq 1.25$ due to resonances with the phonon band.

In order to study the effects of the inhomogeneity on the existence and properties of localized modes, we have performed a continuation from the homogeneous lattice case of $\alpha=0$. We found that, if $\alpha$ increases ( $\alpha>0$, attractive impurity case), the amplitude of the stable on-site mode decreases, while if $\alpha$ decreases ( $\alpha<0$, repulsive impurity case), in general, the stable on-site soliton localized at the impurity merges with the unstable intersite centered one localized between impurity and its neighboring site (beyond some critical value of $|\alpha|$ ) and the resulting state becomes unstable. Notice that, at heart, the latter effect is a pitchfork bifurcation as the on-site mode collides with both the intersite mode centered to its right, as well as with the one centered to its left.

In Fig. 2 we show a typical bifurcation scenario where, for a fixed value of the frequency $\omega$ and the coupling parameter $C$, we depict the mode power $P$ corresponding to different on-site and intersite localized modes as a function of impurity parameter $\alpha$. If we denote as $n_{0}$ the site of the impurity, when $\alpha>0$ increases, we found that the unstable intersite soliton localized at $n=n_{0}+0.5$ disappears in a saddle-node bifurcation with the stable site soliton localized at $n=n_{0}+1$. Also, if we continue this stable mode, when $\alpha$ decreases, and for a given value $\alpha=\alpha_{c}<0$, it also disappears together with the unstable mode localized at $n=n_{0}+1.5$ through a saddle-node bifurcation. If we increase again the impurity parameter, this unstable mode localized at $n=n_{0}$ +1.5 bifurcates with the stable site mode localized at $n=n_{0}$ +2 for a critical value of parameter $\alpha=\alpha_{c}^{\prime}>0$ through a saddle-node bifurcation again, and it could be possible to continue this bifurcation pattern until a site $n_{0}+k$, where the value of site $k$ increases with the value of the coupling $C$ and the frequency $\omega$ parameters. This scenario is similar to the one found in previous studies with different kinds of impurities $[38,44]$ and appears to be quite general. It should be noted that when the coupling parameter increases, more bifurcations take place, in a narrower interval of power $P$ and impurity parameter $\alpha$ values.

Some of the particularly interesting experimentally tractable suggestions that this bifurcation picture brings forward are the following:

(i) A localized mode centered at the impurity may be impossible for sufficiently large attractive impurities (because the amplitude of the mode may decrease to zero), while it may be impossible to observe also in the defocusing case due to the instability induced by the pitchfork bifurcation with its neighboring intersite configurations.

(ii) A localized on-site mode centered at the neighborhood of the impurity should not be possible to localize for sufficiently large impurity strength both in the attractive and in the repulsive impurity case.

We have also performed a more detailed study of the bifurcation between the on-site nonlinear mode centered at the impurity and its intersite and one-site neighbor. Thus, we have determined that, for a given value of the coupling parameter $C$, the corresponding critical value of impurity parameter $\alpha=\alpha_{c}$. Note that this bifurcation takes place only if $\alpha$ is negative (repulsive impurity). In case of $\alpha$ positive (attractive impurity), the intersite solution disappears in a saddle-node bifurcation with the on-site wave centered at the site next to the impurity. In these cases, via an analysis of invariant manifolds of the DNLS map, and following the method developed in Ref. [52] (see the Appendix), some approximate analytical expressions corresponding to this bifurcation point can be obtained. Figure 3 shows the comparison between the exact numerical and the approximate analytical results. In general, for a fixed value of the coupling parameter $C$, the critical value of the frequency increases with $|\alpha|$.

\section{THRESHOLD FOR SOLITARY WAVE FORMATION}

We now examine the problem of solitary wave formation, i.e., whether there exists a minimal, say, amplitude threshold for a compactum of initial data $u_{n}(0)=A \delta_{n, k}$ to nucleate a localized mode. The recent work of Ref. [46] suggests that a good approximation to the amplitude of a single-site initial condition at site $k$ required to nucleate a nonlinear localized mode at that site is given by

$$
-\frac{A^{4}}{2}+\left(2 C-\alpha_{k}\right) A^{2}<0 .
$$

In this expression, $\alpha_{k}$ is the impurity parameter value at site $k$, and $A$ the amplitude of the initial condition [46]. 

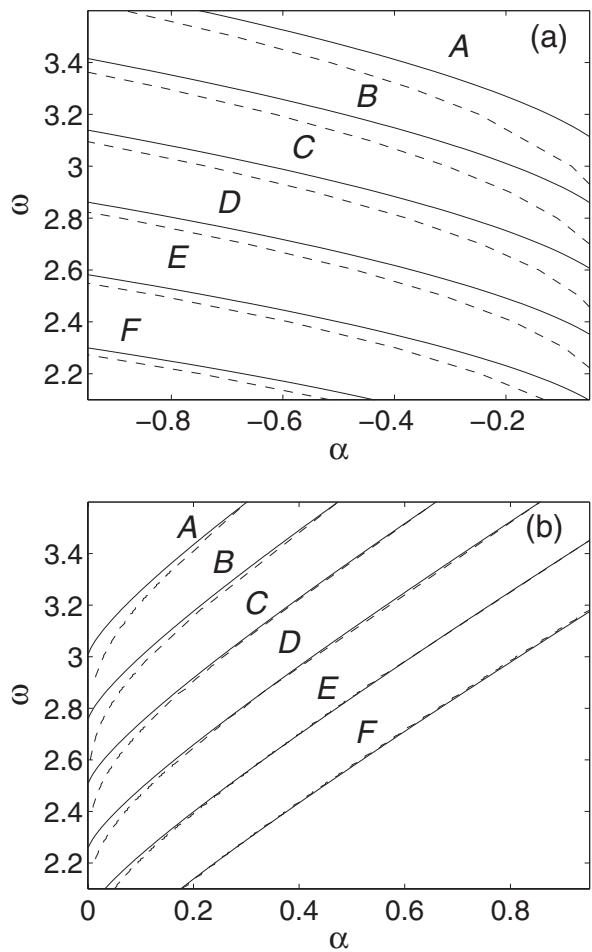

FIG. 3. Bifurcation loci for different values of parameter $C$. (a) The bifurcation between the on-site localized mode at the impurity $\left(n=n_{0}\right)$ and its neighbor intersite breather $\left(n=n_{0}+0.5\right)$, and (b) the bifurcation between the on-site localized mode next to the impurity $\left(n=n_{0}+1\right)$ and its neighbor intersite breather $\left(n=n_{0}+0.5\right)$. Dashed lines correspond to numerical results and continuous lines to approximate analytical calculations. The curve designation is as follows: $A$ corresponds to $C=1.2, B$ to $C=1.1, C$ to $C=1.0, D$ to $C$ $=0.9, E$ to $C=0.8$, and $F$ to $C=0.7$.

In order to study the effect of the impurity on this magnitude, we have performed numerical simulations initially "seeding" energy at different sites of the lattice (either at the impurity or at its neighbors). After a transient state, we have analyzed the existence of localized modes on the chain. To measure the localization of a state we have introduced the localization of an initial excitation of amplitude $A, L(A)$, as

$$
L(A)=\frac{\sum_{n}\left|\varphi_{n}\right|^{2}}{\left(\sum_{n}\left|\varphi_{n}\right|\right)^{2}} .
$$

Thus, for a single excited particle we have $L=1$, and if we have $n$ excited particles (with the same amplitude, and the rest with zero amplitude), $L=1 / n$. In general, $1 / N \leq L \leq 1$.

In Fig. 4 we summarize our numerical results and analytical prediction. In general, when a single perturbation is located on the impurity, numerical and analytical results are in good agreement [Fig. 4(a)]. On the other hand, when the perturbation is located in other (nearby to the impurity) sites of the chain, the excitations of impurity dynamics play a significant role, and numerical and analytical thresholds show a slight divergence [Fig. 4(b)]. However, when the per-
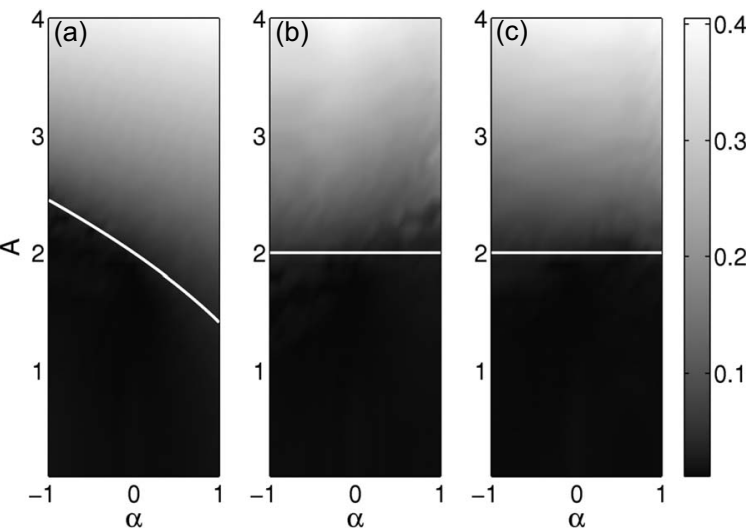

FIG. 4. Localization as function of amplitude $A$ and impurity parameter $\alpha$ for a single excitation $\varphi_{n}(t=0)=A \delta_{n, k}$. (a) The excitation localized at the impurity $\left(k=n_{0}\right)$, (b) the excitation localized at the first neighbor of the impurity $\left(k=n_{0}+1\right)$, while (c) is the second neighbor of the impurity $\left(k=n_{0}+2\right)$. The solid line depicts in each case the theoretical threshold given by Eq. (13). In all cases $N$ $=200$ and $C=1.0$.

turbation is located far enough of the impurity (that for the purposes of formation of a localized mode, we return to the limit of a "homogeneous" lattice), the effect of the impurity is negligible, and the threshold corresponding to homogeneous case is in good agreement with the numerical data, as can be appreciated in Fig. 4(c).

These results also suggest an immediately testable experimental prediction, namely that thresholds such as the ones reported in Ref. [47] (see also Ref. [48] for the defocusing case) should be directly affected by the presence of a localized impurity. In particular, an attractive linear impurity facilitates the formation of localized modes, by decreasing the threshold of their formation, while the opposite is true for repulsive impurities that increase the corresponding threshold.

\section{INTERACTION OF A MOVING LOCALIZED MODE WITH A SINGLE IMPURITY}

Early studies of the DNLS had shown that discrete solitary waves in the DNLS can propagate along the lattice with a relatively small loss of energy [53], and more recent work suggests that such propagating solutions might exist, at least for some range of control parameters [7,54,55]; nevertheless, genuinely traveling solutions are not present in the DNLS, but only in variants of that model (such as the ones with saturable or cubic nonlinearity [56-58]).

In this section we study the interaction of propagating (with only weak radiative losses) localized modes with the impurity. Thus, we consider a nonlinear localized mode, far enough from the impurity, of frequency $\omega$, and perturb it by adding a thrust $q$ to a stationary breather $\phi_{n}$ [59], so that

$$
\varphi_{n}(t=0)=\phi_{n} e^{i q n} .
$$

This is similar in spirit to the examination of Ref. [43], although we presently examine both attractive and repulsive 


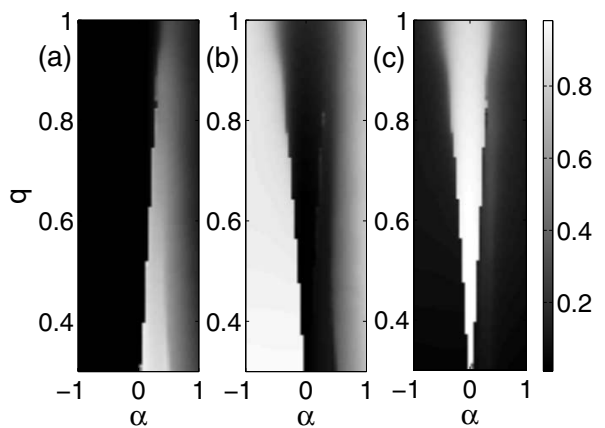

FIG. 5. (a) Power trapping, (b) reflection, and (c) transmission coefficients as function of impurity parameter $\alpha$ and initial thrust $q$. In all cases $N=1000$ and $C=1$.

impurities. In the remainder of this study we consider $\omega$ $=2.5$ and $C=1$, but we have checked that a similar scenario emerges for other values of the frequency $\omega$.

In general, if $q$ is large enough, the soliton moves with a small loss of radiation. We have calculated, as a function of parameters $q$ and $\alpha$, the power and energy that remains trapped by the impurity, reflected and transmitted along the chain, and determined the corresponding coefficients of trapping, reflection, and transmission, defined as the fraction of power (energy) that is trapped, reflected, or transmitted. In Fig. 5 we summarize our results.

We can essentially distinguish the following four fundamental regimes:

(a) Trapping. If the parameters $q$ and $\alpha$ are small enough, and the impurity is attractive, nearly all the energy remains trapped at the impurity, and only a small fraction of energy is lost by means of phonon radiation. An example of this phenomenon is shown in Fig. 6(a). In this case, the central power (power around the impurity) before the collision is nearly zero. When the localized mode reaches the impurity, it loses power as phonon radiation and remains trapped. The analysis of the Fourier spectrum of this trapped breather, carried out after the initial decay and at an early stage of the evolution, shows a frequency close to the initial soliton frequency, as shown in Fig. 6(b). We have observed that, in general, this frequency is slightly smaller than that of the incident soliton, and, in consequence, it has even smaller energy (in absolute value) and power than the corresponding nonlinear mode with the frequency of incident soliton.

In this particular case, corresponding to $q=0.3$ and $\alpha$ $=0.2$, the initial incident wave (after perturbation) has power $P=2.61$ and energy $E=-5.40$ and the stationary mode, trapped at the impurity, with the same frequency, has $P$ $=2.17$ and $E=-4.73$. Thus, the incident breather can activate this nonlinear mode, and nearly all energy and norm remains trapped. In all simulations we have detected similar phenomena, as reported recently in a Klein-Gordon system [60,61].

(b) Trapping and reflection. If the impurity is attractive, but strong enough, some fraction of energy remains trapped by the impurity, but a considerable amount of it is reflected. The reflected excitation remains localized. This case is similar to the previous one, but now the incident traveling structure has enough energy and norm to excite a stationary mode centered at the impurity, remaining localized and give rise to
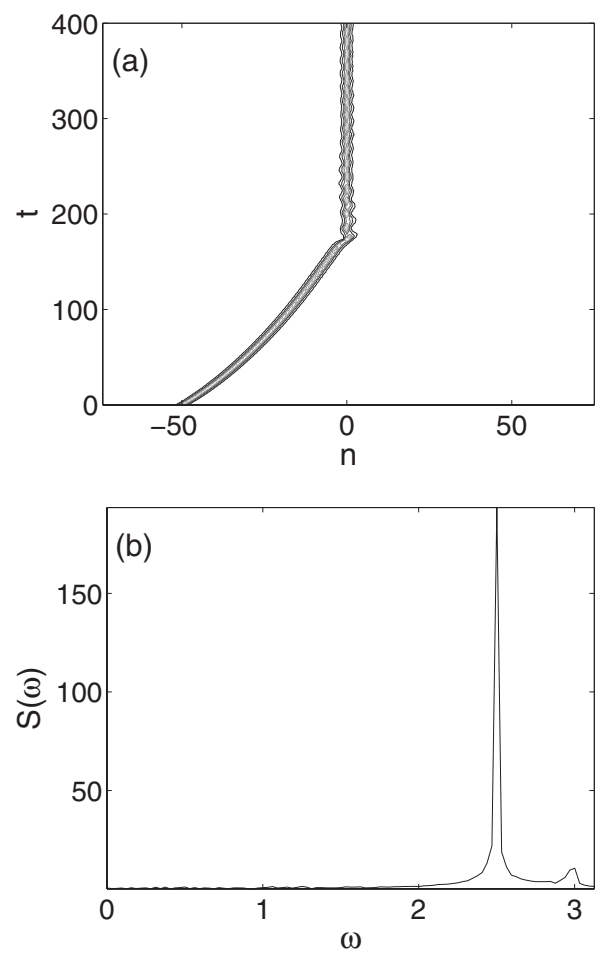

FIG. 6. Trapping: (a) Contour plot corresponding to the power of soliton $P$ as function of site $n$ and time $t$, and (b) Fourier components of the trapped soliton calculated soon after the collision. The parameters are $\alpha=0.2, q=0.3, \omega=2.5, C=1$, and the impurity is located at $n=0$.

a reflected pulse. A typical case is shown in Fig. 7, corresponding to $q=0.6$ and $\alpha=1.0$. The incident wave has power and energy $P=2.61$ and $E=-4.79$, and the stationary nonlinear mode centered at the impurity, with the same frequency, $P=0.76$ and $E=-1.79$. When the incident breather reaches the impurity, it excites the nonlinear mode, and, after losing some energy (in absolute value), part of it remains localized, and another part is reflected. Also, in our numerical simulations, we have detected, as in the previous case, that the frequency of the remaining trapped mode is slightly lower than that of the incident breather, so it has even smaller energy (in absolute value) and power than the corresponding nonlinear mode with the frequency of incident soliton.

In general, we have found that a necessary condition to trap energy and power by the impurity is the existence of a nonlinear localized mode centered at the impurity, with similar frequency, and energy (in absolute value) and power smaller than that of the corresponding incident soliton.

(c) Reflection with no trapping. Here, we have to distinguish two cases. If the impurity is repulsive, and $q$ small enough, neither trapping, nor transmission occur. Instead, all energy is reflected, and the traveling nonlinear excitation remains localized. In this case, as shown in Fig. 8(a), the incident wave has no energy and power to excite the localized mode. In a typical case, i.e., $\omega=2.5, q=0.6$, and $\alpha=-0.5$, the incident soliton has energy and power $E=-4.79$ and $P$ $=2.61$, and the nonlinear localized mode on the impurity with the same frequency $E=-8.038$ and $P=3.77$. No trapping phenomenon occurs, and the pulse is reflected. 

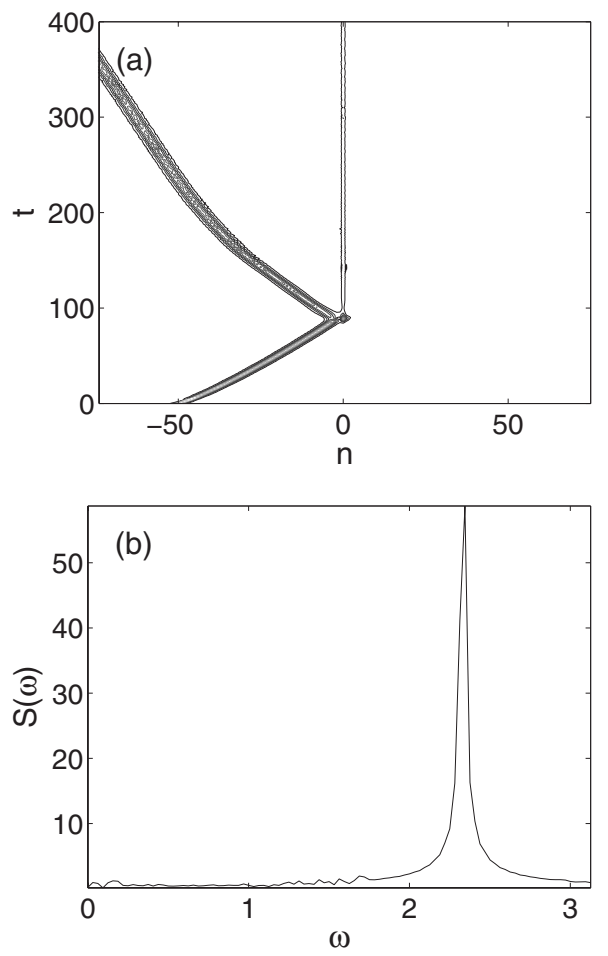

FIG. 7. Trapping and reflection: (a) Contour plot corresponding to the power of soliton $P$ as a function of site $n$ and time $t$, and (b) Fourier components of the trapped soliton calculated soon after the collision. The parameters are $\alpha=1.0, q=0.6, \omega=2.5, C=1$, and the impurity is located at $n=0$.

On the other hand, if the impurity is attractive and strong enough, i.e., $q=0.7, \omega=2.5$, and $\alpha=2.0$, the frequency of the soliton is smaller than the corresponding to linear impurity mode $\left(\omega_{L} \simeq 2.82\right)$, and all the energy is reflected. This is in accordance with the necessity of a nonlinear localized mode at the impurity site in order for the trapping to occur.

(d) Transmission with no trapping. If $|\alpha|$ is small enough, and $q$ high enough, transmission with no trapping occur, as shown in Fig. 8(b). There exists a critical value of $q=q_{c}$ $>0$ that, if $q>q_{c}$, the incident soliton crosses through the impurity. The value of $q_{c}$ grows with $|\alpha|$. In the case where $q<q_{c}$, if $\alpha<0$, reflection with no trapping occurs, while if $\alpha>0$, trapping with no reflection phenomenon takes place.

Our results related to trapping, reflection, and transmission phenomena are in agreement with some results recently obtained, using a different approach, in a similar system [43]. In this work, where approximate discrete moving solitons with fixed amplitude are generated using a continuous approximation, the authors study the trapping process by a linear and a nonlinear attractive impurity. In this latter framework, trapping can be explained by means of resonances with the linear localized mode. In our case, where nonlinear effects become stronger, all this phenomena are related with resonances with a nonlinear localized mode.

Finally, a very interesting phenomenon occurs when parameter $\alpha$ is repulsive and small (in absolute value) enough. In this case, the solitary wave can be reflected or transmitted depending on its velocity. Also, when it is reflected, our numerical tests show that its velocity is similar to its incident
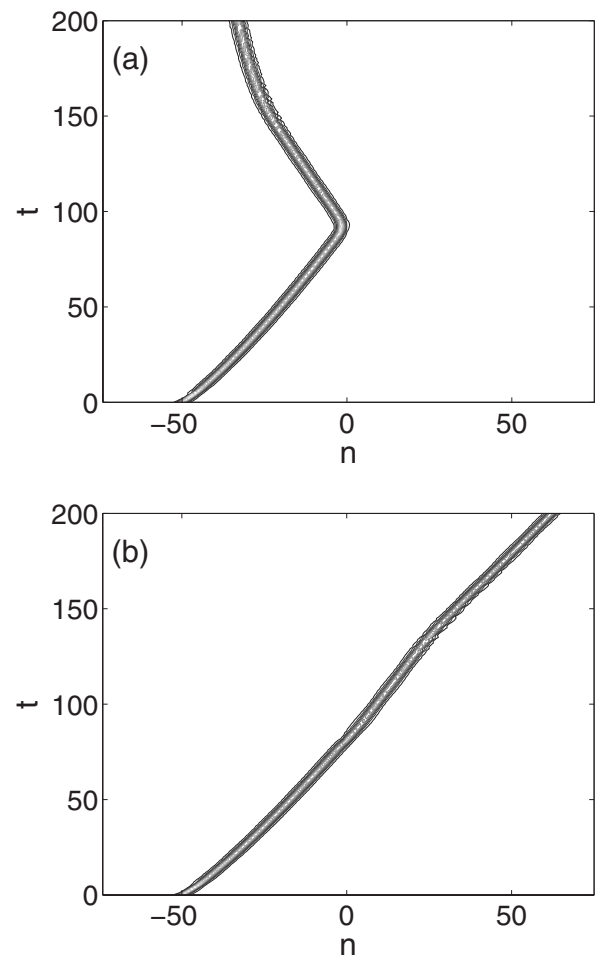

FIG. 8. (a) Reflection with no trapping corresponding to parameters $\alpha=-0.5, q=0.6$, and $\omega=2.5$, and (b) transmission with no trapping corresponding to parameters $\alpha=0.1, q=0.7$, and $\omega=2.5$. In both cases we represent a contour plot corresponding to the power of soliton $P$ as function of site $n$ and time $t, C=1$, and the impurity is located at $n=0$.

velocity. Thus, if we consider the soliton as a "quasiparticle," the effect of the impurity is similar to the effect of a potential barrier. To determine this potential barrier for a given value of parameter $\alpha$, we have used a method similar to the one described by Ref. [62]. We have considered different values of the thrust parameter $q$ corresponding to the reflection regime, and determine, for each value, the turning point, $X(q)$. Thus the translational energy of the barrier for this value of $q$ is defined as the difference between the energy (3) of the moving soliton (15) and the stationary state (5) of the same frequency far from the impurity. It can be written as $V(q)$ $=C \sin (q / 2)|P(q / 2)|$, with $P(q)=i \Sigma_{n} \psi_{n}^{*} \psi_{n+1}-\psi_{n}^{*} \psi_{n-1}$ being the lattice momentum, as defined in Ref. [63]. Results are shown in Fig. 9, which exhibits, as expected, an irregular shape, whose origin lies in the nonuniform behavior of the translational velocity due to the discreteness of the system.

On the other hand, if the parameter $\alpha$ is small enough, and positive (attractive), the solitary wave faces a potential "well" and can be trapped if its translational energy is small or, if the translational energy is high enough, it may be transmitted, losing energy that remains trapped by the impurity, and decreasing its velocity. We have not found a regime with trapping and transmission which has also been observed in Klein-Gordon lattices [60].

\section{CONCLUSIONS}

In this work, we have revisited the long-standing theme of the interactions of DNLS localized modes with an impurity. 

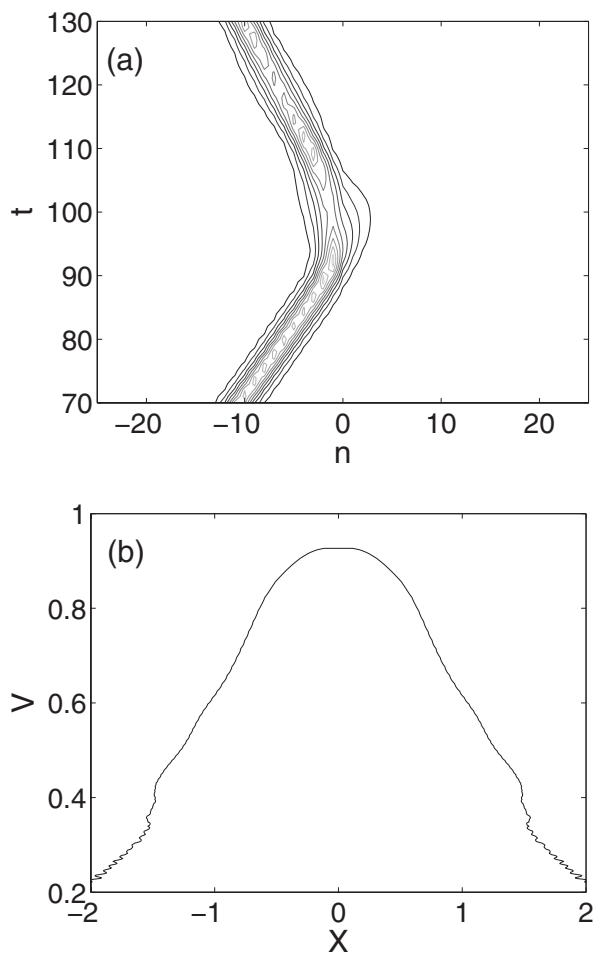

FIG. 9. (a) Contour plot of the phenomenon of reflection of a soliton corresponding to trust parameter $q=0.6$. (b) Potential barrier calculated as described in text. In both cases $\alpha=-0.2, C=1, \omega$ $=2.5$, and the impurity is located at $n=0$.

In particular, we have examined both the case of attractive and repulsive impurities and have shown how localized modes bifurcate out of the linear spectrum in the presence of the impurity. Subsequently, we have seen how drastically the bifurcation diagram of localized modes is affected by the presence of the impurity. In particular, we have concluded that for attractive impurities the on-site mode at the impurity eventually disappears, while for repulsive ones, it becomes unstable beyond a critical impurity strength. In addition, localized modes one site away from the impurity and beyond are also structurally affected and cannot be sustained under strong (either attractive or repulsive) impurities. Furthermore, we have seen how the presence of the impurity significantly modifies the threshold for the formation of localized modes, under a compactum of initial data. Attractive impurities favor the formation of such a mode under weaker excitations, while repulsive ones necessitate an even higher amplitude threshold. Finally, we have examined in detail for both impurity cases (attractive and repulsive) the interaction of the impurity with a moving localized mode initiated away from it. The principal regimes that we have identified as a function of the impurity strength (and sign) and initial speed are trapping, partial trapping, and partial reflection, pure reflection, and pure transmission. In general, if the impurity is repulsive, and the speed small enough, the wave is always reflected. If the impurity strength (in absolute value) is small enough and the speed is high enough, then transmission can take place. On the other hand, if impurity is attractive, trapping can occur, and if the speed is high enough trapping with reflection too. If impurity is attractive and sufficiently strong, the frequency of the soliton is smaller than the one corresponding to the linear localized impurity mode and the wave is reflected.

There are numerous avenues that one can think of for further exploration of this subject. On the one hand, we feel that numerous among the conclusions of the present work including ones about the unavailability of localization on or at nearby sites to the impurity for sufficiently high strengths, or ones about the threshold for localized modes should be immediately experimentally testable in arrays of optical waveguides. On the other hand, this type of wave-impurity interactions have been predominantly studied in onedimensional systems. However, the present availability of two-dimensional waveguide arrays renders this a very interesting system for examining the relevant interaction in multidimensional frameworks, even from a theoretical point of view and the examination of both the standing wave and of the scattering problems. The latter problem is currently under investigation and will be reported in future publications.

\section{ACKNOWLEDGMENTS}

F. P. and J. C. acknowledge financial support from the MECD Project No. FIS2004-01183. F. P. acknowledges San Diego State University for hospitality, and the Secretaría de Estado de Universidades e Investigación del Ministerio de Educación y Ciencia (Spain) for financial support. R. C. G. and P. G. K. gratefully acknowledge support from Contract No. NSF-DMS-0505663. P. G. K. also acknowledges support from Contract No. NSF-DMS-0619492 and NSF-CAREER.

\section{APPENDIX: INVARIANT MANIFOLDS APPROXIMATION}

In this appendix we sketch the method followed in Section 4.1.4 of Ref. [52] for determining the value of $\alpha_{c}$, i.e., the value of $\alpha$ at which the breathers centred at $n=n_{0}$ and $n=n_{0}+0.5$ bifurcate.

The difference equation (6), for $\alpha=0$, can be recast as a two-dimensional real map by defining $y_{n}=\phi_{n}$ and $x_{n}=\phi_{n-1}$ [64],

$$
\left\{\begin{array}{l}
x_{n+1}=y_{n}, \\
y_{n+1}=\left(\omega y_{n}-y_{n}^{3}\right) / C-x_{n} .
\end{array}\right.
$$

For $\omega>2$, the origin $x_{n}=y_{n}=0$ is hyperbolic and a saddle point. Consequently, there exists a one-dimensional stable $\left(W^{s}(0)\right)$ and a one-dimensional unstable $\left(W^{u}(0)\right)$ manifolds emanating from the origin in two directions given by $y$ $=\lambda_{ \pm} x$, with

$$
\lambda_{ \pm}=\frac{\omega \pm \sqrt{\omega^{2}-4 C^{2}}}{2 C} .
$$

These manifolds intersect in general transversally, yielding the existence of an infinity of homoclinic orbits. Each of their intersections corresponds to a localized solution. Fundamental solitons (i.e., on-site and intersite solitons), correspond to the primary intersections points, i.e., those emanating from the first homoclinic windings. Each intersection point defines an initial condition $\left(x_{0}, y_{0}\right)$, that is, $\left(\phi_{-1}, \phi_{0}\right)$, 


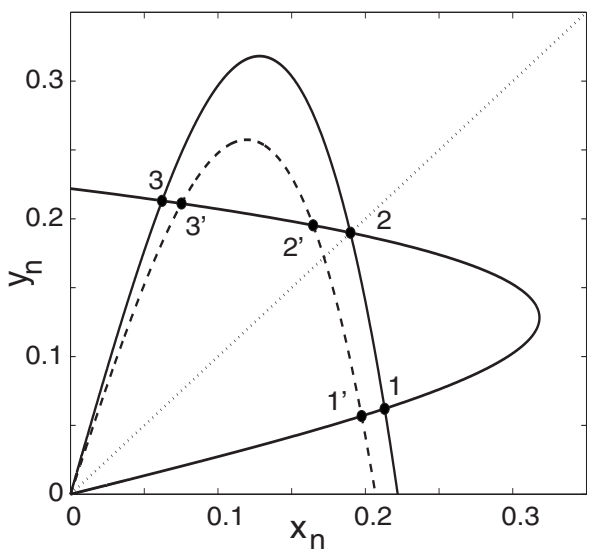

FIG. 10. First winding of the homoclinic tangle of the map (A1). Dashed line corresponds to the linear transformed unstable manifold when $\alpha=0$. Labels $1,2,3\left(1^{\prime}, 2^{\prime}, 3^{\prime}\right)$ corresponds to fundamental solitons for $\alpha=0(\alpha \neq 0)$.

and the rest of the points composing the soliton are determined by application of the map (A1) and its inverse. Figure 10 shows an example of the first windings of the manifolds. Intersections corresponding to fundamental solitons are labeled as follows: (1) is the on-site breather centered at $n=0$, (2) is the intersite breather centered at $n=0.5$, and (3) is the on-site breather centered at $n=1$.

The effect of the inhomogeneity is introduced as a linear transformation of the unstable manifold $A(\alpha) W^{u}(0)$ with $A(\alpha)$ given by

$$
A(\alpha)=\left(\begin{array}{cc}
1 & 0 \\
-\alpha / C & 1
\end{array}\right) .
$$

When $\alpha>0$, the unstable manifold moves downwards, changing the intersections between the transformed unstable manifold and the stable manifold to points $1^{\prime}, 2^{\prime}$, and $3^{\prime}$ (see Fig. 10). For $\alpha=\alpha_{c}$, both manifolds become tangent. Thus, for $\alpha>\alpha_{c}$ intersections $3^{\prime}$ and $2^{\prime}$ are lost, that is, for $\alpha=\alpha_{c}$ the breathers centered at $n=1$ and $n=0.5$ experience a tangent bifurcation. On the contrary, if $\alpha<0$, intersections 1' and $2^{\prime}$ are lost when $|\alpha|>\left|\alpha_{c}\right|$, leading to a bifurcation between the breathers centered at $n=0.5$ and $n=0$.

A method for estimating $\alpha_{c}(\omega)$ is based on a simple approximation of $W^{u}(0)$. Let us consider a cubic approximation
$W_{\text {app }}^{u}$ of the local unstable manifold of Fig. 10, parametrized by $y=\lambda x-c^{2} x^{3}$, with $\lambda \equiv \lambda_{+}$. The coefficient $c$ depends on $\omega$ and $C$ and need not be specified in what follows (a value of $c$ suitable when $\lambda$ is large is computed in Ref. [65]). We have

$$
y=\lambda_{0} x-c^{2} x^{3}
$$

on the curve $A(\omega, \alpha) W_{\text {app }}^{u}$, where $\lambda_{0}=\lambda-\alpha / C$. By symmetry we can approximate the local stable manifold using the curve $W_{\text {app }}^{s}$ parametrized by

$$
x=\lambda y-c^{2} y^{3} .
$$

The curves $A(\alpha) W_{\text {app }}^{u}$ and $W_{\text {app }}^{s}$ become tangent at $(x, y)$ when in addition

$$
\left(\lambda-3 c^{2} x^{2}\right)\left(\lambda_{0}-3 c^{2} y^{2}\right)=1 .
$$

In order to compute $\alpha_{c}$ as a function of $\omega$, or, equivalently, the corresponding value of $\lambda_{0}$ as a function of $\lambda$, one has to solve the nonlinear system (A4)-(A6) with respect to $x, y, \lambda_{0}$, which yields a solution depending on $\lambda$. Instead of using $\lambda$ it is practical to parametrize the solutions by $t=y / x$. This yields

$$
\begin{gathered}
x=\frac{1}{c \sqrt{2}}\left(t+\frac{1}{t^{3}}\right)^{1 / 2}, \quad y=\frac{t}{c \sqrt{2}}\left(t+\frac{1}{t^{3}}\right)^{1 / 2}, \\
\lambda_{0}=\frac{3}{2} t+\frac{1}{2 t^{3}}, \quad \lambda=\frac{3}{2 t}+\frac{1}{2} t^{3} .
\end{gathered}
$$

Since $\lambda+\lambda^{-1}=\omega / C$ it follows that

$$
\begin{aligned}
& t^{4}-2 \lambda t+3=0, \\
& \alpha=\frac{C}{2}\left(t-\frac{1}{t}\right)^{3} .
\end{aligned}
$$

Given a value of $\omega$, one can approximate $\alpha_{c}$ by the value of $\alpha$ given by Eqs. (A7) and (A8). In particular, Eq. (A7) has two real positive solutions (one larger than 1 , and another smaller than 1), and two complex conjugated solutions. The solution with $t>1(t<1)$ leads to $\alpha_{c}>0\left(\alpha_{c}<0\right)$ and, subsequently, approximates the tangent bifurcation values when the breathers at $n=0.5$ and $n=1(n=0)$ collides.

Despite it gives precise numerical results in a certain parameter range, the approximation (A7) and (A8) is not always valid. Indeed, the parameter regime $\omega<5 C / 2$ is not described within this approximation [65].
[1] S. Aubry, Physica D 103, 201 (1997); S. Flach and C. R. Willis, Phys. Rep. 295, 181 (1998); D. Hennig and G. Tsironis, ibid. 307, 333 (1999).

[2] D. N. Christodoulides, F. Lederer, and Y. Silberberg, Nature (London) 424, 817 (2003); Yu. S. Kivshar and G. P. Agrawal, Optical Solitons: From Fibers to Photonic Crystals (Academic Press, San Diego, 2003).

[3] V. A. Brazhnyi and V. V. Konotop, Mod. Phys. Lett. B 18, 627 (2004); O. Morsch and M. Oberthaler, Rev. Mod. Phys. 78,
179 (2006); P. G. Kevrekidis and D. J. Frantzeskakis, Mod. Phys. Lett. B 18, 173 (2004).

[4] M. Sato, B. E. Hubbard, and A. J. Sievers, Rev. Mod. Phys. 78, 137 (2006).

[5] M. Peyrard, Nonlinearity 17, R1 (2004).

[6] P. G. Kevrekidis, K. Ø. Rasmussen, and A. R. Bishop, Int. J. Mod. Phys. B 15, 2833 (2001).

[7] J. C. Eilbeck and M. Johansson, in Localization and Energy Transfer in Nonlinear Systems, edited by L. Vázquez, R. S. 
MacKay, and M. P. Zorzano (World Scientific, Singapore, 2003), pp. 44-67.

[8] H. S. Eisenberg, Y. Silberberg, R. Morandotti, A. R. Boyd, and J. S. Aitchison, Phys. Rev. Lett. 81, 3383 (1998).

[9] R. Morandotti, U. Peschel, J. S. Aitchison, H. S. Eisenberg, and Y. Silberberg, Phys. Rev. Lett. 83, 2726 (1999).

[10] H. S. Eisenberg, Y. Silberberg, R. Morandotti, and J. S. Aitchison, Phys. Rev. Lett. 85, 1863 (2000).

[11] D. Mandelik, R. Morandotti, J. S. Aitchison, and Y. Silberberg, Phys. Rev. Lett. 92, 093904 (2004).

[12] R. Morandotti, H. S. Eisenberg, Y. Silberberg, M. Sorel, and J. S. Aitchison, Phys. Rev. Lett. 86, 3296 (2001).

[13] N. K. Efremidis, S. Sears, D. N. Christodoulides, J. W. Fleischer, and M. Segev, Phys. Rev. E 66, 046602 (2002).

[14] J. W. Fleischer, M. Segev, N. K. Efremidis, and D. N. Christodoulides, Nature (London) 422, 147 (2003); J. W. Fleischer, T. Carmon, M. Segev, N. K. Efremidis, and D. N. Christodoulides, Phys. Rev. Lett. 90, 023902 (2003).

[15] D. Neshev, E. Ostrovskaya, Yu. S. Kivshar, and W. Krolikowski, Opt. Lett. 28, 710 (2003).

[16] H. Martin, E. D. Eugenieva, Z. Chen, and D. N. Christodoulides, Phys. Rev. Lett. 92, 123902 (2004).

[17] J. Yang, I. Makasyuk, A. Bezryadina, and Z. Chen, Opt. Lett. 29, 1662 (2004).

[18] J. Yang, I. Makasyuk, A. Bezryadina, and Z. Chen, Stud. Appl. Math. 113, 389 (2004).

[19] J. Yang, I. Makasyuk, P. G. Kevrekidis, H. Martin, B. A. Malomed, D. J. Frantzeskakis, and X. Zhigang Chen, Phys. Rev. Lett. 94, 113902 (2005).

[20] D. Neshev, Yu. S. Kivshar, H. Martin, and Z. Chen, Opt. Lett. 29, 486 (2004).

[21] D. N. Neshev, T. J. Alexander, E. A. Ostrovskaya, Yu. S. Kivshar, H. Martin, I. Makasyuk, and Z. Chen, Phys. Rev. Lett. 92, 123903 (2004); 92, 123904 (2004).

[22] X. Wang, Z. Chen, and P. G. Kevrekidis, Phys. Rev. Lett. 96, 083904 (2006).

[23] J. W. Fleischer, G. Bartal, O. Cohen, T. Schwartz, O. Manela, B. Freedman, M. Segev, H. Buljan, and N. K. Efremidis, Opt. Express 13, 1780 (2005).

[24] Z. Chen, H. Martin, A. Bezryadina, D. N. Neshev, Yu. S. Kivshar, and D. N. Christodoulides, J. Opt. Soc. Am. B 22, 1395 (2005).

[25] S. Burger, F. S. Cataliotti, C. Fort, P. Maddaloni, F. Minardi, and M. Inguscio, Europhys. Lett. 57, 1 (2002).

[26] A. Smerzi, A. Trombettoni, P. G. Kevrekidis, and A. R. Bishop, Phys. Rev. Lett. 89, 170402 (2002); F. S. Cataliotti, L. Fallani, F. Ferlaino, C. Fort, P. Maddaloni, and M. Inguscio, New J. Phys. 5, 71 (2003).

[27] B. Eiermann, Th. Anker, M. Albiez, M. Taglieber, P. Treutlein, K.-P. Marzlin, and M. K. Oberthaler, Phys. Rev. Lett. 92, 230401 (2004).

[28] M. Jona-Lasinio, O. Morsch, M. Cristiani, N. Malossi, J. H. Müller, E. Courtade, M. Anderlini, and E. Arimondo, Phys. Rev. Lett. 91, 230406 (2003).

[29] B. P. Anderson and M. A. Kasevich, Science 282, 1686 (1998).

[30] See e.g., A. A. Maradudin, Theoretical and Experimental Aspects of the Effects of Point Defects and Disorder on the Vibrations of Crystal (Academic Press, New York, 1966).

[31] See, e.g., I. M. Lifschitz, Nuovo Cimento, Suppl. 3, 716
(1956); I. M. Lifschitz and A. M. Kosevich, Rep. Prog. Phys. 29, 217 (1966).

[32] A. F. Andreev, JETP Lett. 46, 584 (1987); A. V. Balatsky, Nature (London) 403, 717 (2000).

[33] M. I. Molina and G. P. Tsironis, Phys. Rev. B 47, 15330 (1993); G. P. Tsironis, M. I. Molina, and D. Hennig, Phys. Rev. E 50, 2365 (1994).

[34] E. Lidorikis, K. Busch, Q. Li, C. T. Chan, and C. M. Soukoulis, Phys. Rev. B 56, 15090 (1997).

[35] S. Y. Jin, E. Chow, V. Hietala, P. R. Villeneuve, and J. D. Joannopoulos, Science 282, 274 (1998); M. G. Khazhinsky and A. R. McGurn, Phys. Lett. A 237, 175 (1998).

[36] K. Forinash, M. Peyrard, and B. Malomed, Phys. Rev. E 49, 3400 (1994).

[37] W. Krolikowski and Yu. S. Kivshar, J. Opt. Soc. Am. B 13, 876 (1996)

[38] Yu. S. Kivshar, P. G. Kevrekidis, and S. Takeno, Phys. Lett. A 307, 287 (2003).

[39] M. Agrotis, P. G. Kevrekidis, and B. A. Malomed, Math. Comput. Simul. 69, 223 (2005).

[40] S. Longhi, Phys. Rev. E 74, 026602 (2006).

[41] U. Peschel, R. Morandotti, J. S. Aitchison, H. S. Eisenberg, and Y. Silberberg, Appl. Phys. Lett. 75, 1348 (1999).

[42] R. Morandotti, H. S. Eisenberg, D. Mandelik, Y. Silberberg, D. Modotto, M. Sorel, C. R. Stanley, and J. S. Aitchison, Opt. Lett. 28, 834 (2003).

[43] L. Morales-Molina and R. A. Vicencio, Opt. Lett. 31, 966 (2006).

[44] P. G. Kevrekidis, Yu. S. Kivshar, and A. S. Kovalev, Phys. Rev. E 67, 046604 (2003).

[45] E. Smirnov, C. E. Rüter, M. Stepić, V. Shandarov, and D. Kip, Opt. Express 14, 11248 (2006).

[46] P. G. Kevrekidis, J. A. Espinola-Rocha, Y. Drossinos, and A. Stefanov, e-print arXiv:0711.1895.

[47] H. S. Eisenberg, Y. Silberberg, R. Morandotti, A. R. Boyd, and J. S. Aitchison, Phys. Rev. Lett. 81, 3383 (1998).

[48] M. Matuszewski, C. R. Rosberg, D. N. Neshev, A. A. Sukhorukov, A. Mitchell, M. Trippenbach, M. W. Austin, W. Krolikowski, and Yu. S. Kivshar, Opt. Express 14, 254 (2006).

[49] H. Trompeter, U. Peschel, T. Pertsch, F. Lederer, U. Streppel, D. Michaelis, and A. Bräuer, Opt. Express 11, 3404 (2003).

[50] F. Palmero, J. Dorignac, J. C. Eilbeck, and R. A. Römer, Phys. Rev. B 72, 075343 (2005).

[51] J. L. Marín and S. Aubry, Nonlinearity 9, 1501 (1996).

[52] G. James, B. Sánchez-Rey, and J. Cuevas, e-print arXiv:nlin.PS/0710.4114.

[53] J. C. Eilbeck, in Computer Analysis for Life Science Progress and Challenges in Biological and Synthetic Polymer Research, edited by C. Kawabata and A. R. Bishop (Ohmsha, Tokyo, 1986), pp. 12-21.

[54] H. Feddersen, in Nonlinear Coherent Structures in Physics and Biology, edited by M. Remoissenet and M. Peyrard, Lecture Notes in Physics Vol. 393 (Springer, Berlin, 1991), pp. 159167.

[55] J. Gómez-Gardeñes, L. M. Floría, M. Peyrard, and A. P. Bishop, Chaos 14, 1130 (2004).

[56] Y. S. Kivshar and D. K. Campbell, Phys. Rev. E 48, 3077 (1993)

[57] L. Hadžievski, A. Maluckov, M. Stepic, and D. Kip, Phys. Rev. Lett. 93, 033901 (2004). 
[58] T. R. O. Melvin, A. R. Champneys, P. G. Kevrekidis, and J. Cuevas, Phys. Rev. Lett. 97, 124101 (2006); O. F. Oxtoby and I. V. Barashenkov, Phys. Rev. E 76, 036603 (2007).

[59] J. Cuevas and J. C. Eilbeck, Phys. Lett. A 358, 15 (2006).

[60] J. Cuevas, F. Palmero, J. F. R. Archilla, and F. Romero, J. Phys. A 35, 10519 (2002); Theor. Math. Phys. 137, 1406 (2003).

[61] A. Alvarez, F. R. Romero, J. F. R. Archilla, J. Cuevas, and P. V. Larsen, Eur. Phys. J. B 51, 119 (2006).

[62] J. Cuevas and P. G. Kevrekidis, Phys. Rev. E 69, 056609 (2004).

[63] I. E. Papacharalampous, P. G. Kevrekidis, B. A. Malomed, and
D. J. Frantzeskakis, Phys. Rev. E 68, 046604 (2003).

[64] D. Hennig, K. Ø. Rasmussen, H. Gabriel, and A. Bülow, Phys. Rev. E 54, 5788 (1996); T. Bountis, H. W. Capel, M. Kollmann, J. C. Ross, J. M. Bergamin, and J. P. van der Weele, Phys. Lett. A 268, 50 (2000); G. L. Alfimov, V. A. Brazhnyi, and V. V. Konotop, Physica D 194, 127 (2004); R. CarreteroGonzález, J. D. Talley, C. Chong, and B. A. Malomed, ibid. 216, 77 (2006); W. X. Qin and X. Xiao, Nonlinearity 20, 2305 (2007).

[65] J. Cuevas, G. James, B. A. Malomed, P. G. Kevrekidis, and B. Sánchez-Rey, e-print arXiv:nlin.PS/0712.1837. 